\title{
THE NUMBER OF INDECOMPOSABLE SEQUENCES OVER AN ARTIN ALGEBRA OF FINITE TYPE
}

\author{
STEPHEN P. CORWIN
}

(Communicated by Donald Passman)

\begin{abstract}
Let $\Lambda$ be an artin algebra of finite representation type. For a finitely generated $\Lambda$-module $C$, there are only finitely many f.g. modules $A$ such that $0 \rightarrow A \rightarrow B \rightarrow C \rightarrow 0$ is indecomposable as a short exact sequence.
\end{abstract}

Let $\Lambda$ be an artin algebra of finite representation type and $\bmod \Lambda$ the category of finitely generated (f.g.) left $\Lambda$ modules. If $X$ and $C$ are in $\bmod \Lambda$, we write ${ }_{\Lambda}(X, C)$ for $\operatorname{hom}_{\Lambda}(X, C)$ and $P(X, C)$ for the submodule of ${ }_{\Lambda}(X, C)$ comprising those maps $f: X \rightarrow C$ for which there exists a factorization

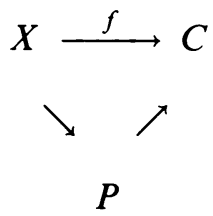

with $P$ projective. Also, let $\operatorname{Tr}$ and $\mathrm{D}$ be the usual transpose and dual. In this setting, Theorem 5.7 in M. Auslander's paper [A] may be stated as follows.

Theorem A. Let $C$ be in $\bmod \Lambda$. Let $A_{1}, \ldots, A_{m}$ be a complete list of all noninjective indecomposable modules in $\bmod \Lambda$ and let $X_{i}=\operatorname{TrD} A_{i}$. For each $i$, ${ }_{\Lambda}\left(X_{i}, C\right) / P\left(X_{i}, C\right)$ is an (End $\left.X_{i}\right)^{\mathrm{op}}$-module of finite length. Let $S_{i_{1}}, \ldots, S_{i_{d_{i}}}$ be a complete set of nonisomorphic simple (End $\left.X_{i}\right)^{\mathrm{op}}$-modules, and for each (End $\left.X_{i}\right)^{\text {op }}$-submodule $H$ of ${ }_{\Lambda}\left(X_{i}, C\right)$ containing $P\left(X_{i}, C\right)$ let $n_{1}\left(A_{i}, H\right), \ldots$, $n_{d_{i}}\left(A_{i}, H\right)$ be the uniquely determined nonnegative integers so that the (End $\left.X_{i}\right)^{\mathrm{op}}$-socle of ${ }_{\Lambda}\left(X_{i}, C\right) / H$ is isomorphic to $\amalg_{j=1}^{d_{i}} S_{i_{j}}^{n_{j}\left(A_{i}, H\right)}$. Finally let $n\left(A_{i}\right)=\max \left\{n_{j}\left(A_{i}, H\right)\right\}$ as $j$ runs through $1,2, \ldots, d_{i}$ and as $H$ runs through all (End $\left.X_{i}\right)^{\text {op }}$-submodules of ${ }_{\Lambda}\left(X_{i}, C\right)$ containing $P\left(X_{i}, C\right)$. Then

(1) $n\left(A_{i}\right)$ is finite;

(2) if $k>n\left(A_{i}\right)$ and $0 \rightarrow A_{i}^{k} \stackrel{g}{\longrightarrow} B \rightarrow C \rightarrow 0$ is exact, then $A_{i}^{k}$ contains a submodule $A^{\prime}$ (isomorphic to $A_{i}^{k-n\left(A_{i}\right)}$ ) such that $g\left(A^{\prime}\right)$ is a summand of $B$.

Received by the editors March 24, 1988.

1980 Mathematics Subject Classification (1985 Revision). Primary 16A64, 16A35, 16A46. 
Keeping the notation of Theorem A we have

Theorem 1. Fix $C$ in $\bmod \Lambda$. Then there are only a finite number of modules $A$ in $\bmod \Lambda$ for which $0 \rightarrow A \stackrel{g}{\longrightarrow} B \rightarrow C \rightarrow 0$ is indecomposable as a short exact sequence. In fact, if $A$ has an injective summand, or if $A \simeq \coprod_{i=1}^{m} A_{i}^{p_{i}}$ with $p_{i}>n\left(A_{i}\right)$ for some $i$, the sequence decomposes.

Proof. If $A$ has an injective summand then clearly the sequence decomposes. Suppose $p_{i}>n\left(A_{i}\right)$, and form the pushout diagram

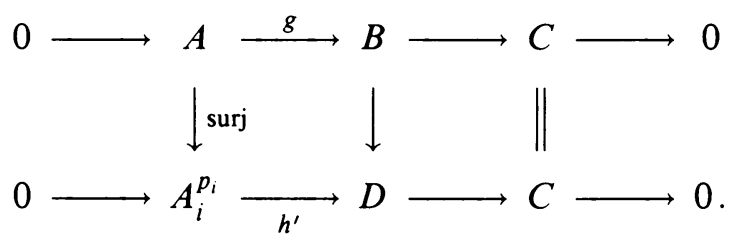

Because $p_{i}>n\left(A_{i}\right), A_{i}^{p_{i}}$ has a submodule $A^{\prime}$ for which $h^{\prime}\left(A^{\prime}\right)$ is a summand of $D$ (so $A^{\prime}$ is actually a summand of $A_{i}^{p_{i}}$ ) by Theorem A. Let $A^{\prime \prime}, A^{\prime \prime \prime}$ be such that $A^{\prime} \oplus A^{\prime \prime}=A$ and $A^{\prime} \oplus A^{\prime \prime \prime}=A_{i}^{p_{i}}$. Then we have a commutative diagram

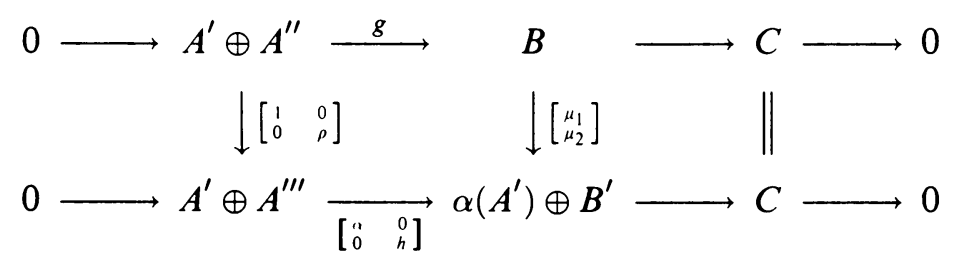

in which $\alpha=\left.h^{\prime}\right|_{A^{\prime}}$ is an isomorphism and $h=\left.h^{\prime}\right|_{A^{\prime \prime \prime}}$. Then $A^{\prime} \stackrel{1}{\longrightarrow} A^{\prime} \stackrel{g}{\longrightarrow}$ $g\left(A^{\prime}\right)$ is a monomorphism which is split by $B \stackrel{\mu_{1}}{\longrightarrow} \alpha\left(A^{\prime}\right) \stackrel{\alpha^{-1}}{\longrightarrow} A^{\prime}$, so $g\left(A^{\prime}\right)$ is a summand of $B$. This split monomorphism and the split inclusion of $A^{\prime}$ into $A$ are coherent, i.e., the diagram

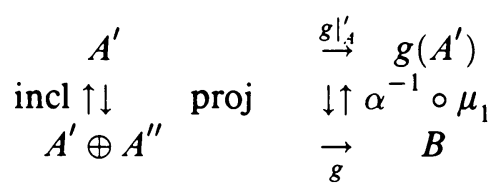

commutes both ways. Thus the exact sequence $0 \rightarrow A^{\prime} \stackrel{\left.g\right|_{A} ^{\prime}}{\longrightarrow} g\left(A^{\prime}\right) \rightarrow 0 \rightarrow 0$ is a summand of $0 \rightarrow A \rightarrow B \rightarrow C \rightarrow 0$.

If we let $R$ be a local PID which is also a $k$-algebra, we get some interesting consequences. We let $f$ and $g$ be matrices over $R$, and say that $X=F_{2} \stackrel{f}{\longrightarrow}$ $F_{1} \stackrel{g}{\longrightarrow} F_{0}$ is a representation of the diagram $A_{2}=\cdot \rightarrow \cdot \rightarrow \cdot$ over $R$, where $F_{2}, F_{1}$, and $F_{0}$ are free $R$-modules (see, e.g., [DR]). If $f$ and $g$ are both 
$t \times t$ matrices with nonzero determinant, then the sequence $\varepsilon=0 \rightarrow A \rightarrow B \rightarrow$ $C \rightarrow 0$ is naturally associated with $X$, where $A=\operatorname{coker}(f), B=\operatorname{coker}(g f)$, and $C=\operatorname{coker}(g)$, by the following commutative diagram:

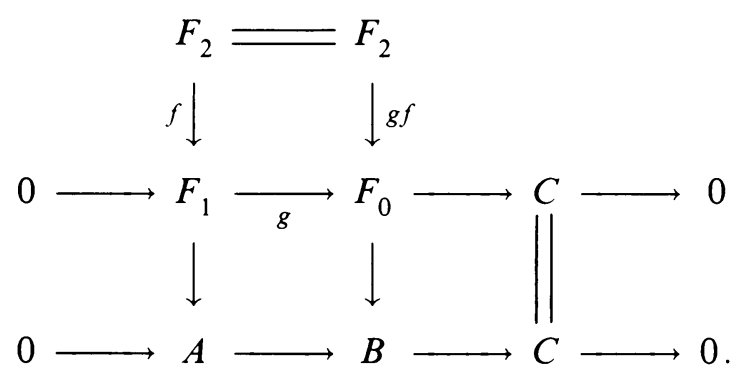

Two representations $X, X^{\prime}$ are said to be isomorphic if there is a commutative diagram

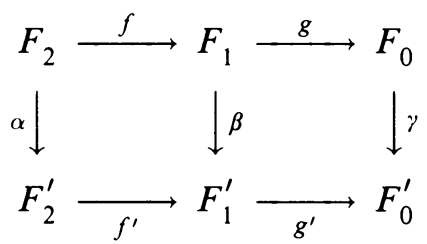

with $\alpha, \beta$, and $\gamma$ isomorphisms. It is shown in [C] that representations are isomorphic if and only if the corresponding sequences are isomorphic.

If $\underline{m}$ is the maximal ideal of $R$ and $f$ is a $t \times t$ matrix, we let $\nu(f)$ be the least integer $n$ such that $\operatorname{det}(f) \in \underline{m}^{n}$ (where $\underline{m}^{0}$ is the set of units of $R$ ). In this situation Theorem 1 yields the following.

Corollary. Let $g$ be a fixed $t \times t$ matrix with nonzero determinant, and let $\nu(g)=r$. Then for a fixed integer $n$, there are only finitely many nonisomorphic indecomposable representations $X=F_{2} \stackrel{f}{\longrightarrow} F_{2} \stackrel{g}{\longrightarrow} F_{0}$ with $\nu(f) \leq n$.

Proof. If $0 \rightarrow A \rightarrow B \rightarrow C \rightarrow 0$ is the sequence associated with $X$, then the length of an indecomposable summand of $C$ (respectively $A$ ) is bounded by $r$ (respectively $n$ ); so every such sequence may be considered to be a sequence of $R / \underline{m}^{s}$-modules, where $s=\max \{r, n\}$. But $R / \underline{m}^{s}$ is an artin algebra of finite type, so Theorem 1 may be applied.

An application of this corollary, proved in [C], is that if $\nu(f)<t$, where $t$ is as above, then $X$ must decompose.

\section{ACKNOWLEDGMENT}

The author would like to thank E. L. Green, who supervised the dissertation in which these results were first obtained. 


\section{REFERENCES}

[A] M. Auslander, Functors and morphisms determined by objects (R. Gordon, ed.), Lecture Notes Pure Appl. Math., no. 37, Marcel Dekker, New York, 1976.

[C] S. P. Corwin, Representation theory of the diagram $A_{n}$ over the ring $k[[x]]$, Dissertation, Virginia Tech, Blacksburg, Virginia.

[DR] V. Dlab and C. M. Ringel, Indecomposable representations of graphs and algebras, Mem. Amer. Math. Soc., no. 173, 1976.

Department of Mathematics, Radford University, Radford, Virginia 24142 